\title{
SENP1 has an important role in lung development and influences the differentiation of alveolar type 2 cells
}

\author{
XUE-QING WAN, JIA-YU CAI, YUE ZHU, QIU-XIA WANG, HAI-TAO ZHU, HUI-MIN JU and HONG-YAN LU \\ Department of Pediatrics, Affiliated Hospital of Jiangsu University, Zhenjiang, Jiangsu 212000, P.R. China
}

Received June 16, 2018; Accepted October 22, 2018

DOI: $10.3892 / \mathrm{ijmm} .2018 .3964$

\begin{abstract}
Post-translational modification via small ubiquitin-like modifier (SUMO) is involved in the regulation of various important cellular processes. SUMO modification can be regulated at the level of conjugation, and can also be reversed by the SUMO-specific proteases (SENPs). However, current studies of the regulation and function of SENP in lung development remain limited. In this study, the expression levels of SENP1 and SUMO1 were assessed during lung development in rats. SUMO1 modification occurred during lung development and changes in SENP1 expression were consistent with the changes in the presence of free SUMO1. In order to investigate the function of SENP1, alveolar type (AT) 2 cells were transfected with SENP1-targeting small interfering RNA, and the proliferation, apoptosis and differentiation function of AT2 cells was subsequently evaluated. Marked upregulation of conjugated SUMO1 was observed following SENP1 inhibition. Furthermore, depletion of SENP1 resulted in increased apoptosis, decreased proliferation and impaired differentiation status of AT2 cells. Thus, the results support that SENP1 is an essential regulator of the balance between SUMOylation and deSUMOylation during lung development, specifically affecting the proliferation and differentiation status of AT2 cells.
\end{abstract}

\section{Introduction}

Lung development is a highly conserved process that can be broken down into four phases: Origination of the lung anlage; formation of the trachea; morphogenesis of branches; and finally, alveologenesis and reduction in cell proliferation with the emergence of more differentiated cell types (1). In the late stage of lung development, alveolar airspaces are formed.

Correspondence to: Professor Hong-Yan Lu, Department of Pediatrics, Affiliated Hospital of Jiangsu University, 438 Jiefang Road, Zhenjiang, Jiangsu 212000, P.R. China

E-mail: 1hy5154@163.com

Key words: small ubiquitin-like modifier 1, SUMO-specific proteases, SUMOylation, lung development, alveolar type 2 cells, differentiation
These are the principal units involved in gas exchange (2). Lung alveolar epithelial cells are comprised of alveolar type (AT) 1 and AT2. AT2 cells serve as progenitors for AT1 cells during development and cell damage (3-5). This late stage of lung development requires highly coordinated regulation; any slight disturbance in alveolarization may lead to severe diseases or developmental disorders (6).

Small ubiquitin-like modifier (SUMO) conjugation to proteins is a reversible post-translational modification that participates in various cellular and developmental processes (7). In mammals, there are three main SUMO protein subtypes: SUMO1, SUMO2 and SUMO3. Due to their high sequence homology, SUMO2 and SUMO3 are usually referred as SUMO2/3. SUMO1 is conjugated to proteins even under normal physiological conditions (8). SUMO2/3 are usually found unconjugated, but rapidly bind to proteins in several stress conditions (9). Covalent attachment of SUMO to proteins is assisted by the E1 activating enzyme, E2 conjugating enzyme (Ubc9) and E3 ligase. Deconjugation from targets is regulated by SUMO specific proteases (SENPs). In addition to deSUMOylation, SENPs also catalyze the maturation of SUMO precursor. Thus, SENPs are a key regulatory mechanism for maintaining a SUMO balance. Six SENPs (SENP1, 2, 3, 5, 6 and 7) are present in humans and mice. Out of these six proteins, SENP1 has been the most widely studied. SENP1 is involved in various pathophysiological processes, including transcriptional regulation, cell proliferation, differentiation and development (10-12). Current studies indicate that disorder in either SUMO conjugation or deconjugation can contribute to embryonic lethality, chromosomal defects, abnormal nuclear morphology and promote the occurrence of diseases $(13,14)$. Ubc9 deficiency in mice leads to embryonic lethality at the early developmental stage (15). Mutation of SENP1 in mice causes an increase in SUMO1 conjugation and phenotypic defects in the placenta (16). Experimental data of Ubc9 deficiency and SENP1 mutation indicate that the maintenance of the SUMO cycle is essential for normal growth and development.

In the respiratory system, it has been previously reported that the CCAAT/enhancer-binding protein $\alpha(\mathrm{C} / \mathrm{EBP} \alpha)$ is modified by SUMO1 to mediate lung growth and differentiation (17). SENP1 enhances the proliferative ability of pulmonary artery smooth muscle cells (18). In addition, during the pathogenesis of lung disease, SUMO1 may participate in the modulation of hypoxia-inducible factor-1 $\alpha$ through 
SUMOylation in hypoxic pulmonary hypertension (19). Hypoxia triggers deSUMOylation of Kruppel-like factor 15 by SENP1 and transcriptional regulation of arginase 2 in lung endothelium (20). SENP1 was reported to be overexpressed in lung cancer tissues, while modulation of SENP1 expression was demonstrated to significantly affect the proliferation of lung cancer cells (21). However, little attention has been devoted to the role of SENP1 in the process of lung differentiation. To clarify this process, the expression levels of SUMO1 and SENP1 were examined in rat lung tissue in the current study. The results indicated that SENP1 regulates deSUMOylation of SUMO1-modified proteins during the alveolar development period. It was also demonstrated that SENP1 is required for the differentiation of AT2 cells. Together, these results reveal that SENP1 is involved in lung development and differentiation.

\section{Materials and methods}

Animals and tissue preparation. A total of 32 Sprague-Dawley neonatal rats (weight $6.2 \pm 0.3 \mathrm{~g}$ ) were purchased from the Animal Center of Jiangsu University (Zhenjiang, China). The rats were randomly assigned to 4 groups with 8 rats in each group. Each group of neonatal rats was fed by a 3-month-old female rat. Rats were kept on a 12-h light/dark cycle at a room temperature of $23 \pm 2^{\circ} \mathrm{C}$ and supplied with sufficient food and water. Neonatal rats were sacrificed via cervical dislocation, followed by a sternotomy. Phosphate buffered saline (PBS) was gently perfused into the trachea with an injector to clear the lung tissue. Lungs were collected and frozen immediately for further study at postnatal day 1, 4, 7 and 14 (P1, P4, P7 and P14). All animal research was approved by the Animal Center of Jiangsu University.

Histological analysis. Tissues were fixed with $4 \%$ paraformaldehyde for $24 \mathrm{~h}$ at $4^{\circ} \mathrm{C}$ and washed with PBS. Subsequently, samples were dehydrated using an alcohol gradient (75\% alcohol, $1.5 \mathrm{~h} ; 95 \%$ alcohol, $1.5 \mathrm{~h} ; 100 \%$ alcohol, $1.5 \mathrm{~h} ; 100 \%$ alcohol, $1 \mathrm{~h}$; two xylene washes, $0.5 \mathrm{~h}$ each) and embedded in paraffin. Sections were sliced at $3 \mu \mathrm{m}$, followed by conventional dewaxing in water. Antigen retrieval was performed in $10 \mathrm{mM}$ citrate buffer (pH 6.0) and boiled for $20 \mathrm{~min}$. The tissue sections were stained with hematoxylin and eosin (Solarbio Science \& Technology Co., Ltd., Beijing, China) 2-3 min each for histological analysis. All steps were performed at a room temperature. The sections were acquired by confocal light microscopy (Olympus Corporation, Tokyo, Japan) at magnification of $\mathrm{x} 400$.

Reverse transcription-quantitative polymerase chain reaction (RT-qPCR) for lung tissue. RT-qPCR was used to detect the mRNA expression of SUMO1 and SENP1 at each time-point. Total RNA was isolated from the tissue samples using TRIzol (Invitrogen; Thermo Fisher Scientific, Inc., Waltham, MA, USA). For RT, cDNA was synthesized using the Prime Script RT reagent kit (Takara Biotechnology Co., Ltd., Dalian, China) according to the manufacturer's instructions. qPCR was performed using the SYBR Premix Ex Taq (Takara Biotechnology Co., Ltd., Dalian, China). The reaction conditions were as follows: $95^{\circ} \mathrm{C}$ for $30 \mathrm{sec}$, and 40 cycles of $95^{\circ} \mathrm{C}$ for $5 \mathrm{sec}$ and $60^{\circ} \mathrm{C}$ for $30 \mathrm{sec}$. Target sequences of SUMO1, SENP1 and $\beta$-actin were as follows: SUMO1, 5'-AAGTTA TTGGACAGGACAGCA-3' and 5'-CATTCCCAGTTCTTT TGGAG-3'; SENP1, 5'-CGCCAGATTGAAGAGCAGA-3' and 5'-AGAGGAACACGAAGGTGGAG-3'; $\beta$-actin, 5'-TGT CACCAACTGGGACGATA-3' and 5'-GGGGTGTTGAAG GTCTCAAA-3'. The specificity of the PCR product was ensured by melting curve analysis using the LightCycler ${ }^{\circledR} 96$ (Roche Diagnostics, Basel, Switzerland). All calculations of the relative expression of the target gene were performed using the $2^{-\Delta \Delta \mathrm{Cq}}$ method (22).

Cell culture and grouping. For the experiments, human primary type II alveolar epithelial cells (AT2; cat. no. HUM-iCELL-a002; purchased from iCell Bioscience, Inc., Shanghai, China) were cultured in Dulbecco's modified Eagle's medium/F12 (DME/F-12; HyClone; GE Healthcare Life Sciences, Logan, UT, USA), supplemented with $10 \%$ fetal bovine serum (Wisent, Inc., St. Bruno, QC, Canada), in a $5 \% \mathrm{CO}_{2}$ at $37^{\circ} \mathrm{C}$. For AT2 differentiation, retinoic acid (RA) was added to medium at $1 \mu \mathrm{M}$ (23). RA powder was purchased from Target Molecule Corp. (Boston, MA, USA) and dissolved in dimethyl sulfoxide. To minimize the effect of AT2 cells transforming into AT1 in vitro and ensure stable growth, the primary AT2 cells were passaged for three generations prior used for differentiation. After reaching 80-90\% confluency, the cells were divided into normal control group (NC group), RA group (with $1 \mu \mathrm{M}$ RA in the medium), si-NS group, si-SENP1 group, RA + si-NS group, and RA + si-SENP1 group. The si-SENP1 cells and si-NS cells were transfected with SENP1 small interfering RNA (siRNA) or non-specific (NS) siRNA.

Protein extraction and western blotting. All protein extraction handling was performed on ice. Free SUMO1 and SUMOylated proteins were analyzed. As described by Sharma et al (24), individual lung tissue protein lysates were prepared either using $4 \%$ sodium dodecyl sulfate (SDS) or 1\% Nonident P40 (NP40). SDS denatures the action of SENPs and preserves conjugated SUMO. Therefore, the measured free SUMO1 is the naturally existing free unconjugated SUMO1 protein. NP40 separates SUMO1 from the target. Thus, the measured free SUMO1 represents total SUMO1 including unconjugated and separated SUMO1 in lung tissue. Free SUMO1 and SUMOylated proteins were extracted by $4 \%$ SDS, unless otherwise indicated. Protein extraction for SENP1 detection was performed as described. AT2 cells were harvested using the radioimmunoprecipitation assay buffer containing protease inhibitor phenylmethanesulfonyl fluoride (Sigma-Aldrich; Merck KGaA, Darmstadt, Germany) for cell lysis. The extract was centrifuged at $12,000 \times \mathrm{g}, 4^{\circ} \mathrm{C}$ for $15 \mathrm{~min}$ and the supernatant was collected. The protein concentration was detected using a bicinchoninic acid kit (Beyotime Institute of Biotechnology, Haimen, China). Protein extracts $(10 \mu \mathrm{l})$ were fractionated by $8-16 \%$ SDS-PAGE (GenScript, Piscataway, NJ, USA) and transferred to polyvinylidene difluoride membranes. The membranes were blocked in 5\% milk-Tris-buffered saline (TBS) buffer containing $0.1 \%$ Tween- 20 at $37^{\circ} \mathrm{C}$ for $1 \mathrm{~h}$, then incubated overnight at $4^{\circ} \mathrm{C}$ with primary antibodies. Primary antibodies were applied at dilutions of 1:200 for SENP1 (cat. no. HPA011765; 
Sigma-Aldrich; Merck KGaA), 1:1,000 for surfactant protein C (SP-C; cat. no. sc-13979; Santa Cruz Biotechnology, Inc., Dallas, TX, USA), 1:1,000 for aquaporin-5 (AQP5; cat. no. sc-514022; Santa Cruz Biotechnology, Inc.), 1:1,000 for SUMO1 and 1:1,000 for $\beta$-actin (cat. no. 4903 and cat. no. 3700 from Cell Signaling Technology, Inc., Danvers, MA, USA). Following washing with TBS buffer-0.1\% Tween-20, horseradish peroxidase-coupled secondary antibodies $(1: 2,000$; cat. nos. FMS-MS01, FMS-Rb01 and FMS-Gt01) from Fcmacs Biotech Co., Ltd. (Nanjing, China) were added for $1 \mathrm{~h}$ at $37^{\circ} \mathrm{C}$. The signals were visualized using FluorChem FC3 chemiluminescence (ProteinSimple, San Jose, CA, USA). Due to the significant difference in the protein expression quantity of free SUMO1 and conjugated SUMO1, the exposure time of conjugated SUMO1 (20 sec) was shorter than that of free SUMO1 $(50 \mathrm{sec})$. Thus, they are typically shown separately in immunoblot bands $(24,25)$. Semi-quantitative analysis was performed using LANE 1D software (Beijing Sage Creation Science Co., Ltd., Beijing, China). The target protein and $\beta$-actin were analyzed, and the expression of the target protein at each time-point was compared with with the corresponding internal reference. Statistical analysis was conducted to estimate the relative expression of the target proteins.

Cell differentiation and immunofluorescence. Retinoic acid (RA) is a major bioactive metabolite of vitamin A. It regulates normal lung development and maturation, and preserves alveolar formation in a caloric restriction model $(26,27)$. To investigate the effect of RA on AT2 differentiation, $6 \times 10^{5}$ cells were seeded in $2 \mathrm{ml}$ medium. At a density of $1 \times 10^{7}$ cells per 6-cm culture dish, AT2 cells were randomly divided into negative control (NC) group and RA group (with $1 \mu \mathrm{M}$ RA added to the medium). Cells were harvested at 24,48 and $72 \mathrm{~h}$. Double immunofluorescent staining was used to observe the expression of SP-C and AQP5. AT2 cells were seeded in 24-well plates at a density of $1 \times 10^{4}$ cells/well. The cells were separated into NC group and RA group. Cells were allowed to grow for 24, 48 and $72 \mathrm{~h}$, then fixed with $4 \%$ paraformaldehyde at room temperature for $20 \mathrm{~min}$ and blocked in $3 \%$ fetal calf serum at $37^{\circ} \mathrm{C}$ for $1 \mathrm{~h}$. Primary antibodies against SP-C (1:200; cat. no. sc-13979) and AQP5 (1:200, cat. no. sc-514022; Santa Cruz Biotechnology, Inc.) were incubated overnight at $4^{\circ} \mathrm{C}$. Anti-SP-C was detected using a secondary fluorescein isothiocyanate (FITC)-conjugated antibody (goat anti-rabbit IgG; cat. no. A22120; 1:200; Abbkine Scientific Co., Ltd., Wuhan, China) and anti-AQP5 was detected using a secondary Dylight 594-conjugated antibody (goat anti-mouse IgG; cat. no. ab96873; 1:200; Abcam, Cambridge, MA, USA) with incubated for $1 \mathrm{~h}$ at $37^{\circ} \mathrm{C}$. Cell nuclei were stained with DAPI $(1: 1,000)$ for $3 \mathrm{~min}$ at a room temperature of $23 \pm 2^{\circ} \mathrm{C}$. Fluorescent images were obtained using a fluorescent microscope (Olympus Corporation). Quantification of expression levels of SP-C and AQP5 in fluorescent images was performed using Image-Pro Plus III (Media Cybernetics, Inc., Rockville, MD, USA). The percentage of SP-C or AQP5 is the expression level of SP-C or AQP5/(SP-C+AQP5 expression level).

Cell transient transfection and transfection based on $R A$. Transfections were performed using Lipofectamine ${ }^{\circledR} 2000$
(Invitrogen; Thermo Fisher Scientific, Inc.), in accordance with the manufacturer's instructions, when the cells reached $33 \%$ confluency. To verify the transfection efficiency, AT2 cells were divided into si-NS group and si-SENP1 group with no RA added in medium. To investigate the suppression impact of SENP1 on differentiation, cells were transfected with a SENP1 siRNA or NS siRNA with RA added in the medium. The concentration of each siRNA used for transfection was $50 \mathrm{nM}$. Cells were harvested after transfection for 24, 48 and $72 \mathrm{~h}$. The transfection efficiency of SENP1 was linked to the resulting mRNA and protein expression levels of conjugated SUMO1. The suppressive influence of SENP1 with RA added was detected by RT-qPCR and western blot analysis. Protein and gene expression levels of SP-C and AQP5 were measured to analyze the effects on differentiation. The siRNA sequences were as follows: SENP1 siRNA, 5'-GCC UGACCAUUACACGCAATT-3' and 5'-UUGCGUGUAAUG GUCAGGCTT-3'; NS siRNA: 5'-UUCUCCGAACGUGUC ACGUTT-3' and 5'-ACGUGACACGUUCGGAGAATT-3'. Primer sequences for detecting SP-C, AQP5 and $\beta$-actin were as follows: SP-C, 5'-TTACCACTGCCACCTTCTCC-3' and 5'-TCAAGACTGGGGATGCTCTC-3'; AQP5, 5'-ACTGGG TTTTCTGGGTAGGG-3' and 5'-GTGGTCAGCTCCATG GTCTT-3'; $\beta$-actin, 5'-UGACCUCAACUACAUGGUUTT-3' and 5'-AACCAUGUAGUUGAGGUCATT-3'. The RT-qPCR processes were performed as described.

Cell proliferation. Cell counting kit-8 (CCK-8; Biosharp, Hefei, China) was used to analyze cell proliferation. AT2 cells were randomly into si-NS group and si-SENP1 group. They were then cultured in DME/F-12 for 24, 48 and $72 \mathrm{~h}$. The cells were then seeded in a 96-well plate at a density of 5,000 cells/well in $100 \mu \mathrm{l}$ medium and $10 \mu \mathrm{l} \mathrm{CCK-8;} \mathrm{four}$ duplicate wells were set for each sample. After $2 \mathrm{~h}$ in the cell incubator, and ELISA reader (Bio-Rad Laboratories, Inc., Hercules, CA, USA) was used to measure absorbance at $450 \mathrm{~nm}$.

Cell apoptosis. An Annexin V/propidium iodide (PI)-FITC apoptosis detection kit I (BD Biosciences, Franklin Lakes, NJ, USA) was used to analyze cell apoptosis by flow cytometry. AT2 cells were plated at a density of $6 \times 10^{5}$ cells per $6-\mathrm{cm}$ culture dish, and divided into si-NS and si-SENP1 groups. Cells were harvested after transfection for 24, 48 and $72 \mathrm{~h}$. Cells and medium were collected in a $15 \mathrm{ml}$ centrifuge tube. Trypsinization without EDTA was used to suspend cells. Samples were handled according to the manufacturer's instructions of the apoptosis detection kit. The apoptosis rate of cells in each group was analyzed using an Accuri C6 flow cytometer (CANTO 10C; BD Bioscience).

Statistical analysis. Data are expressed as the mean \pm standard deviation and analyzed using SPSS 17.0 statistical software (SPSS, Inc., Chicago, IL, USA). One-way analysis of variance was used to analyze data differences among multiple groups, and then comparisons between groups were assessed using a Student-Newman-Keuls test. Student's t-test was used to analyze the difference between groups. $\mathrm{P}<0.05$ was considered to indicate a statistically significant difference. 


\section{A}
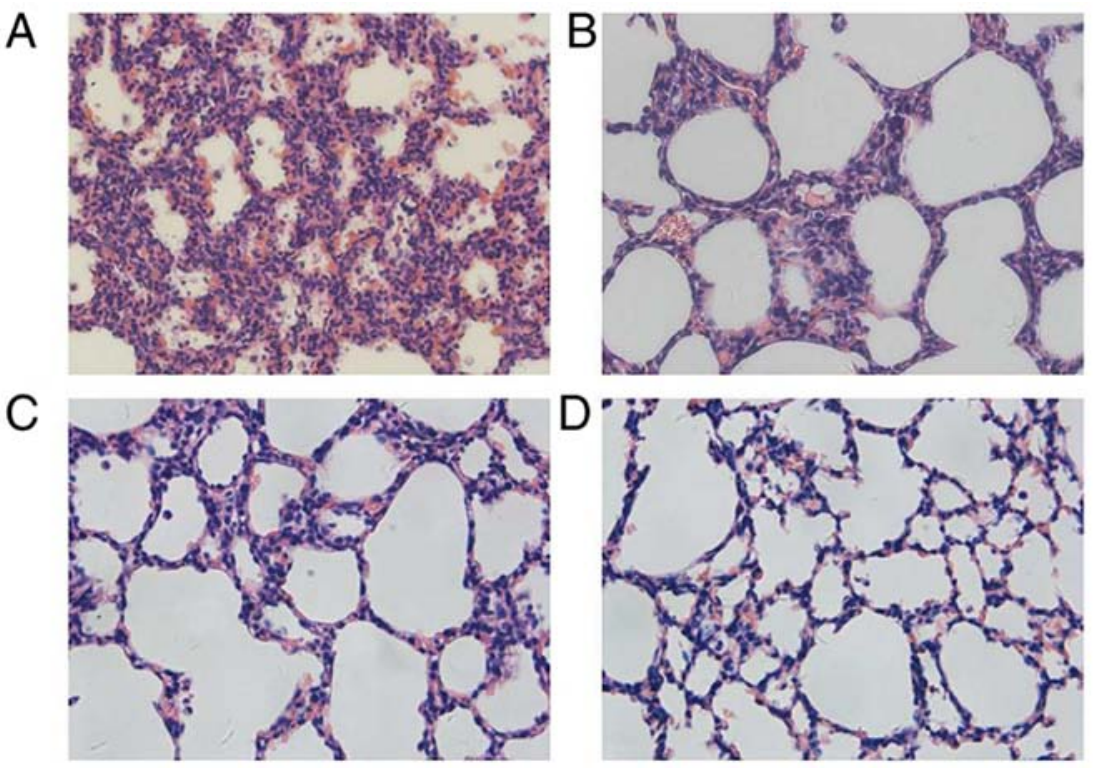

Figure 1. Histology of transverse sections of isolated lungs from several stages of development. Sections were stained with hematoxylin and eosin. (A) Lung section from P1; a large number of primary alveoli, with irregular structures. (B) Lung section from P4; several ridges protruding into the alveolar space. (C) Lung section from P7; air sacs tend to be uniform, and the interstitial tissues thin out. (D) Lung section from P14; mature alveoli is the basic unit in the lung, with uniform size and separated by very thin septum. Magnification, $x 400$.

\section{Results}

Histomorphological variations of lung morphogenesis at different stages. As presented in Fig. 1, irregularity in the structures could be observed in the primary alveoli at P1. At P4, the late canalicular stage, an increasing number of alveolar septa were formed with some ridges protruding into the alveolar space. At P7, the size of air sacs tended to be homogeneous and the interstitial tissues thinned out. By P14, mature alveoli were the basic unit in the lung, appeared uniformly sized and separated by a thin septum.

Lung development proceeds with changes in free SUMO1, but no changes in total SUMO1. To investigate the role of SUMOylation in lung development, whether the process involves changes in the levels of SUMO1 conjugation was determined. Neonatal rats 14 days after birth were used. At this age, their lungs are in the saccular and alveolar period, an important phase of lung maturation. Initially, the protein levels of naturally existing free SUMO1 were detected. As shown in Fig. 2A, free SUMO1 increased at day 4 compared with day 1 , visibly decreased at day 7 compared with day 4, and then expressed at a similar level until day 14. Day 4 and day 14 were selected as time-points for detecting the degree of SUMO conjugation. In western blot analysis, free SUMO1 and bands of higher molecular mass $(>70 \mathrm{kDa})$ were detected indicating SUMOylated proteins (Fig. 2B). Free SUMO1 prepared using 4\% SDS (which represented naturally existing free SUMO1) was compared with free SUMO1 prepared using $1 \%$ NP40 (which represented total SUMO1). Naturally existing free SUMO1 at day 14 exhibited a sharp decrease compared with day 4 , while no significant changes were observed in total SUMO1 (Fig. 2B). The results indicate that the total SUMO1 was steadily expressed and the SUMOylation and deSUMOylation were maintained in a dynamic balance.
SUMO1 mRNA expression is stable, while SENPI is differentially expressed during lung development. Gene expression analysis was performed to further analyze the role of SUMO1 in lung development. RT-qPCR results indicated that SUMO1 mRNA was not significantly changed during lung development from P1 to P4 (Fig. 3A). This indicates that mRNA expression of SUMO1 was maintained in at a stable level. Subsequently, gene expression analysis of SENP1 was performed (Fig. 3B). The results demonstrated an increase in SENP1 mRNA at P4 compared with P1, which decreased at P7 and was maintained a steady level from P7 to P14. To confirm if varied gene expression is consistent with protein expression, SENP1 was also detected using western blot analysis. As presented in Fig. 4, the gene and protein levels of SENP1 exhibited a similar trend during lung development. However, compared with the induction of SENP1 mRNA at $\mathrm{P} 4$, the change in protein expression level was marginal. This difference may be associated with the post-translational modifications, and that protein translation is slightly delayed compared with mRNA transcription. This confirmed that SENP1 may impact lung development.

Retinoic acid promotes AT2 differentiation into AT1 with changes in SENP1. In a previous study, tritium $\left[{ }^{3} \mathrm{H}\right]$ was injected into pregnant rats and the fetal rats were examined (28). This experiment revealed that AT2 cells could convert to AT1 cells during lung development. According to the previous study in rats, during the 14 days after birth, the expression of SP-C was gradually reduced, whereas the expression of AQP5 increased (17,29). SP-C and AQP5 are specific markers of AT2 and AT1 cells, respectively $(30,31)$. This indicated that AT2 differentiation to AT1 may occur during this period. To determine whether SENP1 affects lung development by participating in the differentiation of AT2, AT2 cells were cultured in vitro in the current study and RA was used to 

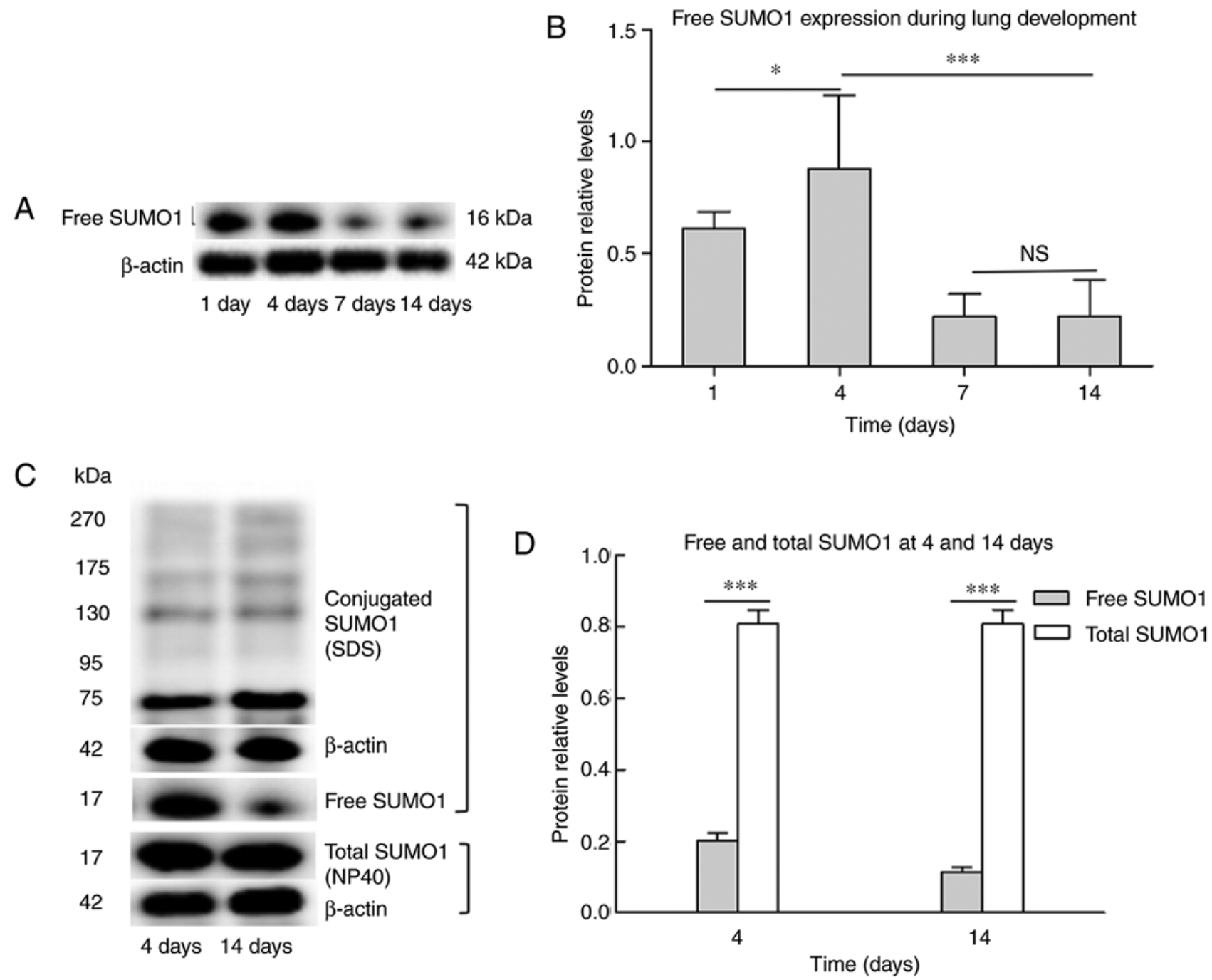

Figure 2. Lung development and associated changes in free SUMO pools. (A) Western blot analysis of the protein expression levels of the naturally existing free SUMO1 in lung tissue extracts of neonatal rats at day 1, 4, 7 and 14. (B) Relative level of free SUMO1 protein as quantified from (A). Free SUMO1 increased at day 4, visibly decreased at day 7 and stably expressed until day 14. Data are presented as the mean \pm standard deviation. (C) For analysis of free SUMO1 and total SUMO1, extracts were prepared either under denaturing conditions (SDS) or under non-denaturing conditions (NP40). (D) Quantification of the free and total SUMO1 expression of the analysis in (C). Decreases in free SUMO1 levels were quantified from day 4 to 14 of lung development. No significant change was observed in total SUMO1. Statistical significance of free SUMO1 with respect to total SUMO1 was analyzed by Student's t-test. Values are presented as the mean \pm standard deviation. ${ }^{*} \mathrm{P}<0.05,{ }^{* * * *} \mathrm{P}<0.001$. Total protein extracts $(10 \mu \mathrm{l})$ were loaded per lane. SUMO, small ubiquitin-like modifier; NS, no significant difference; SDS, sodium dodecyl sulfate; NP40, nonident P40.
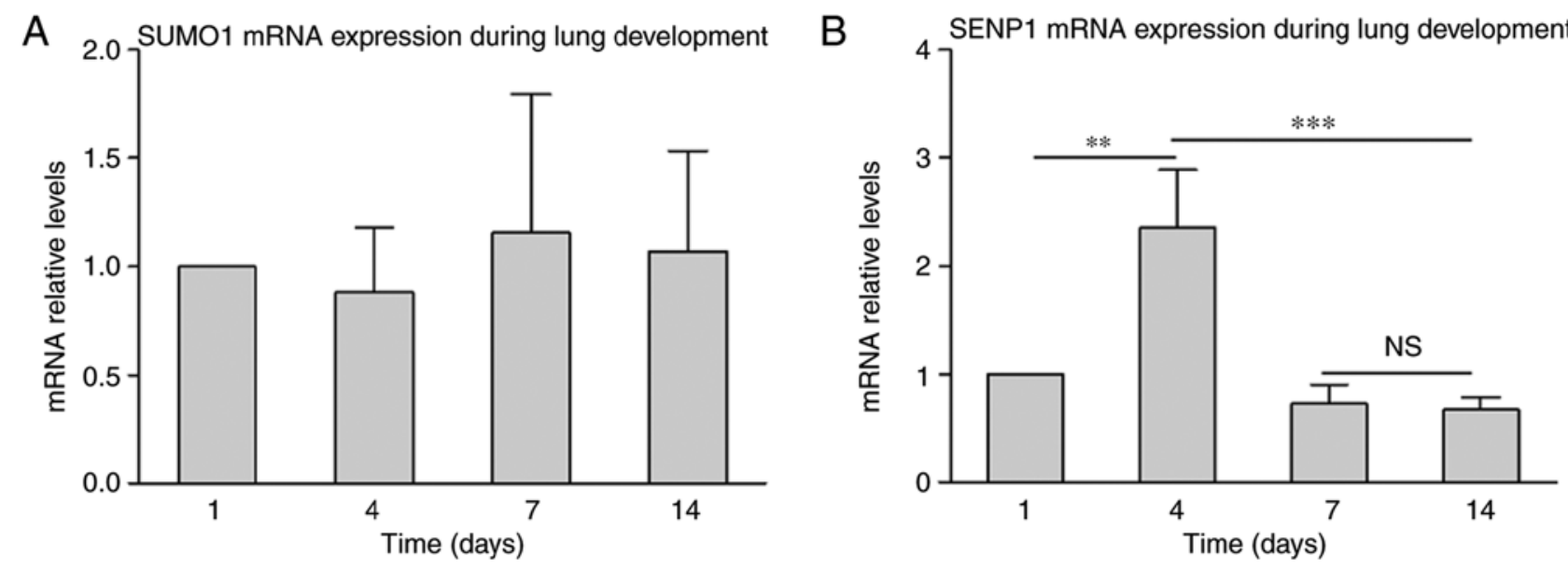

Figure 3. SUMO1 and SENP1 mRNA expression in neonatal rats. Relative expression levels of (A) SUMO1 and (B) SENP1 mRNA were detected by reverse transcription-quantitative polymerase chain reaction. Levels were normalized to the level at day 1 . Values are presented as the mean \pm standard deviation of three independent experiments in duplicate. ${ }^{* *} \mathrm{P}<0.01,{ }^{* * * *} \mathrm{P}<0.001$. SUMO, small ubiquitin-like modifier; SENP1, SUMO specific protease 1; NS, no significant difference. 
A

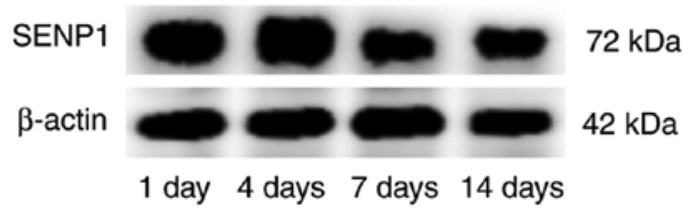

B

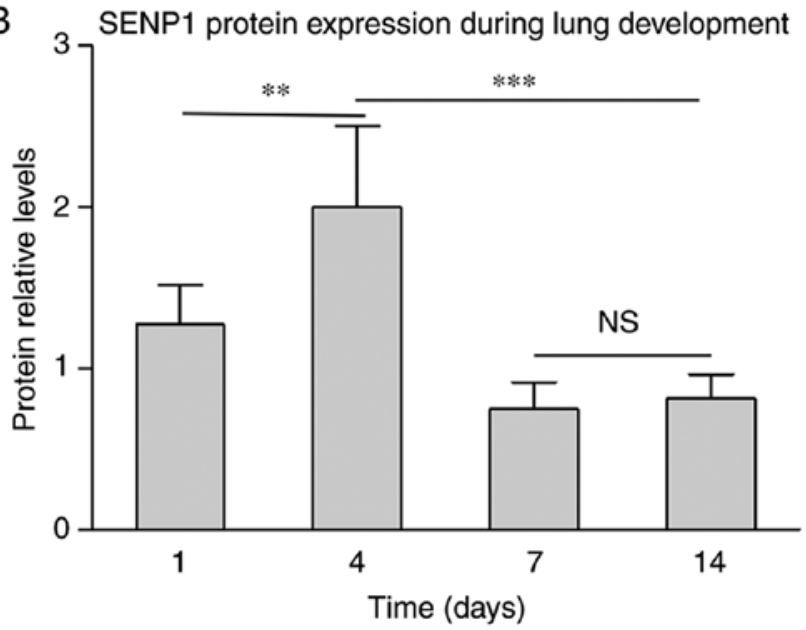

Figure 4. (A) SENP1 protein exhibits a differential expression levels during lung development. Protein levels were analyzed by western blotting. (B) Relative levels of SENP1 protein were semi-quantified. Values are presented as the mean \pm standard deviation. Total protein extracts $(10 \mu \mathrm{l})$ were loaded per lane. Statistical significance was determined using one-way analysis of variance followed Student-Newman-Keuls test, ${ }^{* *} \mathrm{P}<0.01$, ${ }^{* * * *} \mathrm{P}<0.001$. SENP1, SUMO specific protease 1; NS, no significant difference.

promote differentiation. Initially, the differentiation efficiency of RA was examined. AT2 cells were exposed to $1 \mu \mathrm{M} \mathrm{RA}$ and the expression levels of SP-C and AQP5 were then detected using western blot analysis. As shown in Fig. 5A-C, the expression of SP-C was decreased at 24 and $72 \mathrm{~h}$ following treatment with RA. However, it increased was $48 \mathrm{~h}$, which may have been caused by cell proliferation. RA enhanced the expression of AQP5 at 24, 48 and $72 \mathrm{~h}$ compared with untreated cells (Fig. 5C). As shown in Fig. 5A and D, SENP1 protein was increased over time in the control and RA groups. Additionally, the expression of SENP1 in the RA group was increased compared with the control group at 24,48 and $72 \mathrm{~h}$. To validate the changes in SP-C and AQP5, the expression were also measured by immunofluorescence (Fig. 6A and B). These results also demonstrated that the decrease in SP-C was accompanied by an increase in AQP5. The experiments demonstrated that RA promoted the differentiation of AT2. Taken together, these results suggest that SENP1 may participate in the differentiation of AT2.

Inhibition of SENP1 results in conjugated SUMO1 changes and impairs AT2 differentiation. siRNA was used to inhibit SENP1 expression in AT2. Cells were transfected with si-SENP1 and non-specific RNA (si-NS). Transfection efficiency was demonstrated by RT-qPCR and western blot analysis. The results indicated that the gene expression of SENP1 was decreased at 24, 48 and $72 \mathrm{~h}$ after transfection compared with si-NS. SENP1 was decreased 8.8 -fold at $48 \mathrm{~h}$ compared with the corresponding control group, indicating efficient inhibition (Fig. 7A). As SENP1 is a SUMO-specific protease, mediating protein deSUMOylation is its main role. Changes in SUMOylated proteins were detected when SENP1 was depleted using siRNA. The level of conjugated SUMO1 increased sharply, while free SUMO1 was decreased by SENP1 knockdown compared with si-NS (Fig. 7B). This result indicates that the inhibition of SENP1 expression interfered with deSUMOlation. The effect of SENP1 inhibition on cell differentiation was investigated further. AT2 cells were cultured in medium containing RA, then transfected with si-NS (RA + si-NS group) or si-SENP1 (RA + si-SENP1 group). Western blot analysis demonstrated that the expression of SENP1 protein decreased significantly in the RA + si-SENP1 group compared to the RA + si-NS group (Fig. 8A and B), with a 1.5 -fold decrease in protein levels at $24 \mathrm{~h}, 3.2$-fold at $48 \mathrm{~h}$, and 1.6-fold at $72 \mathrm{~h}$. The inhibition efficiency was the most pronounced at $48 \mathrm{~h}$, in accordance with the changes in mRNA expression. The protein expression levels of SP-C and AQP5 were also assessed using western blot analysis. As shown in Fig. 8C and D, compared to the control group, the inhibition of SENP1 decreased the protein level of AQP5 expression at 24,48 and $72 \mathrm{~h}$. The protein expression of SP-C was increased at 24 and $48 \mathrm{~h}$, but decreased at $72 \mathrm{~h}$. The decrease of SP-C may have been caused by growth inhibition. The change in SUMOylated proteins was also detected. As shown in Fig. 8E, the conjugated SUMO1 was increased by si-SENP1 and free SUMO1 was decreased, which was similar to the result with no RA was added (Fig. 7B). The expression level of free and total SUMO1 under these two conditions revealed no statistically significant difference. These results indicate that the inhibition efficiency of SENP1 on conjugated and free SUMO1 has not been affected by the effect of RA on the differentiation of AT2 cells. To determine the effect of SENP1 on cell differentiation, SP-C and AQP5 levels were also analyzed by RT-qPCR. SP-C and AQP5 mRNA expression levels exhibited a similar expression pattern to the relevant protein levels following SENP1 knockdown (Fig. 9). These results indicate that inhibition of SENP1 hinders the differentiation of AT2, and that SENP1 is an important regulator of the differentiation process.

Depletion of SENP1 inhibits proliferation and promotes apoptosis of AT2 cells. SENP1 has an important role in regulating the proliferation and apoptosis of cells. CCK- 8 was used to evaluate the AT2 proliferation in cells transfected with SENP1 siRNA for 24, 48 and $72 \mathrm{~h}$. As shown in Fig. 10A, cell proliferation was reduced in the si-SENP1 group compared with si-NS $(\mathrm{P}<0.05)$. To examine whether SENP1 knockdown also affects apoptosis, flow cytometry analysis and an Annexin V-FITC/PI apoptosis detection kit was used. Early apoptosis rate $(\mathrm{Q} 3)$ and late apoptosis rate $(\mathrm{Q} 2)$ were combined as the total apoptosis rate. SENP1 inhibition promoted cell apoptosis when AT2 cells were transfected with si-SENP1 for 24,48 and $72 \mathrm{~h}$ (Fig. 10B). Following transfection with si-SENP1 for $24 \mathrm{~h}$, the percentage of cells in the early apoptosis subgroup was significantly higher than in the si-NS group $(7.230 \pm 0.816$ vs. $4.573 \pm 0.398 \%$ ); however, SENP1 inhibition had no influence on the late apoptosis. The percentage of cells in both the early and late apoptosis subgroups were higher compared with the si-NS group $(12.833 \pm 1.266$ vs. $7.317 \pm 0.831 \%$; and $7.453 \pm 0.479$ vs. $5.183 \pm 0.629 \%$, respectively) when SENP1 inhibition was increased to $48 \mathrm{~h}$. Furthermore, the percentage of cells in the 

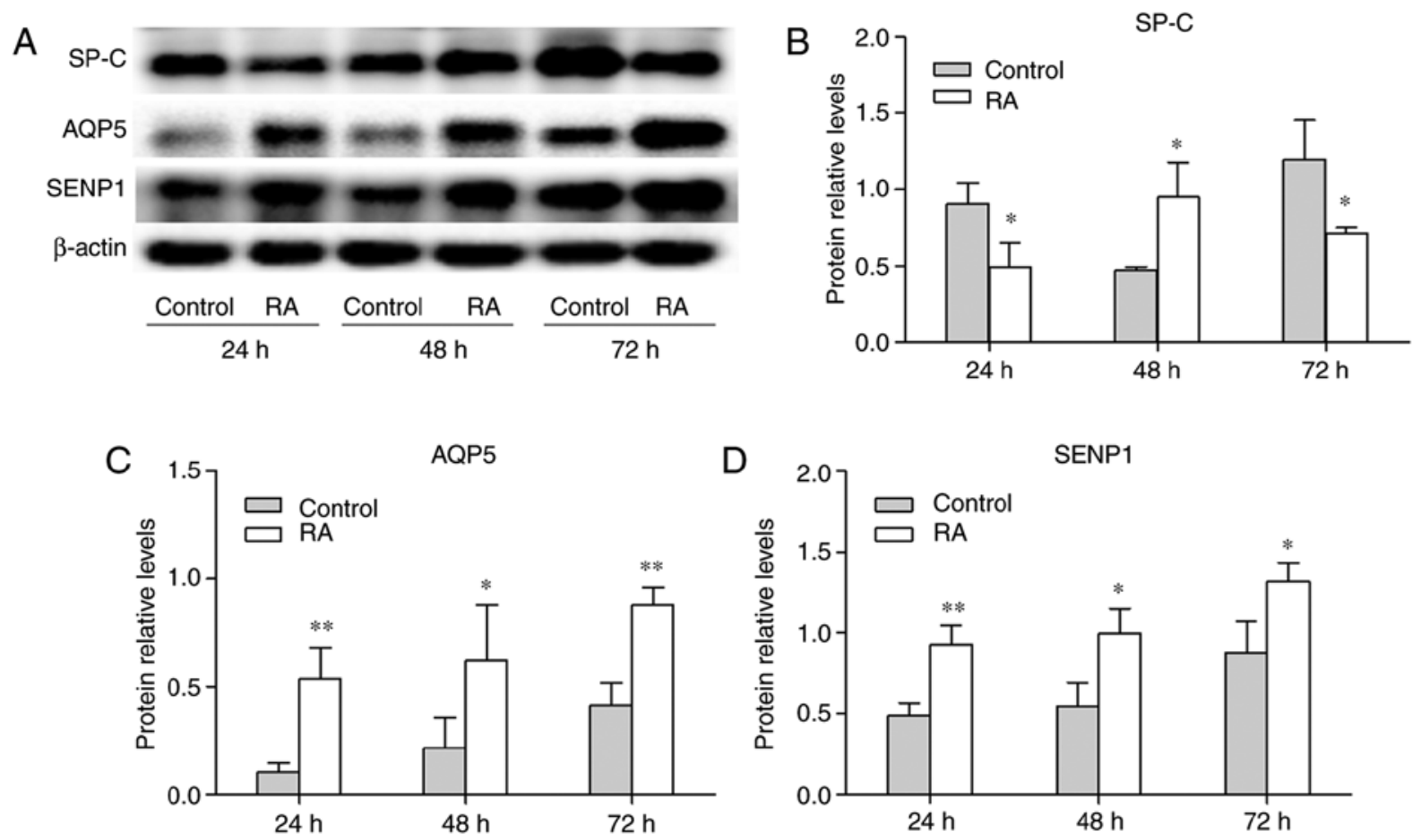

Figure 5. Effect of RA on cell differentiation and SENP1 expression level. (A) Representative western blots of target proteins. The expression levels of (B) SP-C, (C) AQP5 and (D) SENP1 were visualized. Values are presented as the mean \pm standard deviation from three independent experiments. Statistical significance was determined using Student's t-test, ${ }^{*} \mathrm{P}<0.05,{ }^{* *} \mathrm{P}<0.01$. RA, retinoic acid; SP-C, surfactant protein C; AQP5, aquaporin-5; SENP1, SUMO specific protease 1.
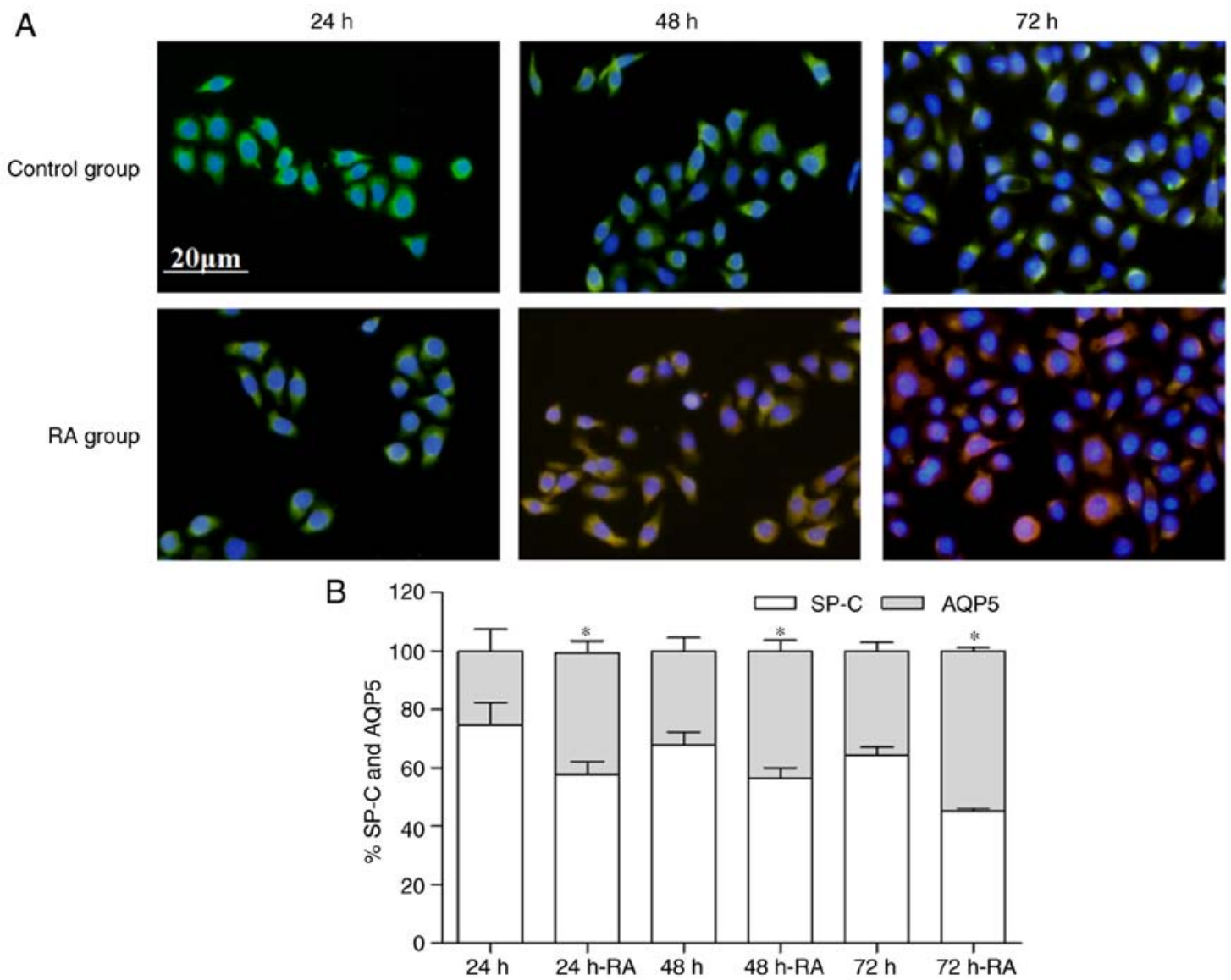

Figure 6. RA induces AT2 differentiation. (A) AT2 cells differentiating to AT1 cells were detected by expression of the respective marker SP-C (green fluorescence) and AQP5 (red fluorescence). Nuclei were counterstained with DAPI (blue fluorescence). Scale bar, $20 \mu \mathrm{m}$. (B) Quantification of the percentage of SP-C and AQP5 as the proportion of AT2 cells differentiating to AT1 cells. The percentage of SP-C or AQP5 is the expression level of SP-C or AQP5/(SP-C+AQP5 expression level). Values are presented as the mean \pm standard deviation, obtained by counting the average optical density from three independent experiments. ${ }^{*} \mathrm{P}<0.05$ vs. control group at the same time-point. RA, retinoic acid; SP-C, surfactant protein C; AQP5, aquaporin-5; SENP1, SUMO specific protease 1. 
A

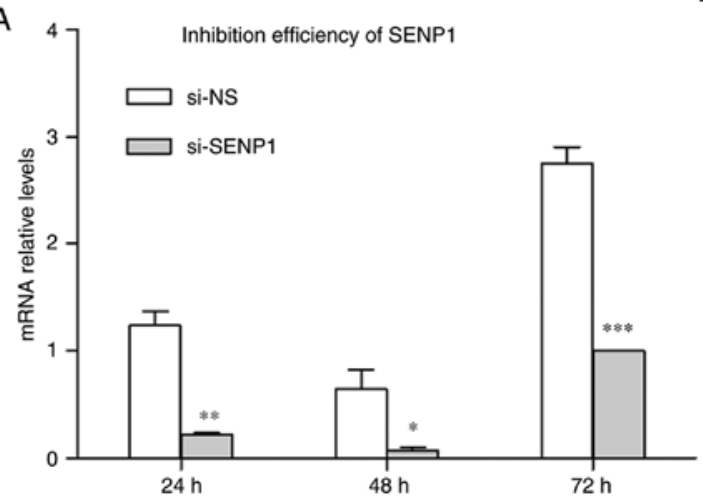

B $\mathrm{kDa}$

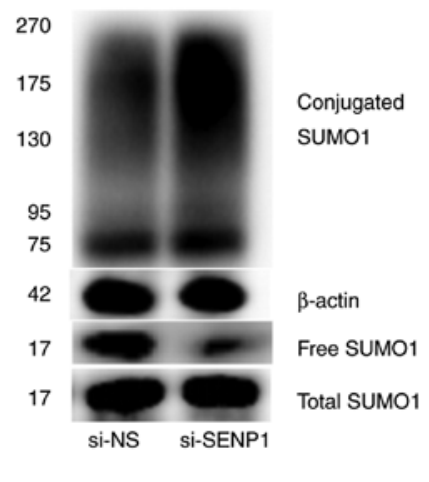

Figure 7. SENP1 inhibition results in upregulation of conjugated SUMO1. (A) Relative expression levels of the SENP1 gene were determined by RT-qPCR. Levels were normalized to the level at $72 \mathrm{~h}$ in the si-SENP1 group. Values are presented as the mean \pm standard deviation of three independent experiments in duplicate. Statistical significance was determined using Student's t-test, ${ }^{*} \mathrm{P}<0.05,{ }^{* *} \mathrm{P}<0.01,{ }^{* * *} \mathrm{P}<0.001$ vs. si-NS group at the same time-point. (B) Protein levels of SUMO1 with SENP1 knockdown were analyzed by western blotting. Protein extracts were prepared under denaturing conditions (sodium dodecyl sulfate). In comparison with the si-NS group, conjugated SUMO1 in the si-SENP1 group was significantly increased. A similar result was observed in RA + si-NS and RA + si-SENP1 group. RA, retinoic acid; SENP1, SUMO specific protease 1; si, small interfering RNA; NS, non-specific; SUMO, small ubiquitin-like modifier.

A

A SPC

AQP5

SENP1

$\beta$-actin

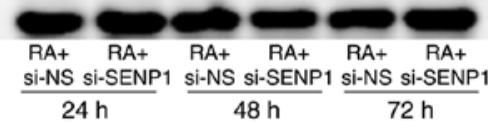

B $\quad 2.0 \quad$ SENP1

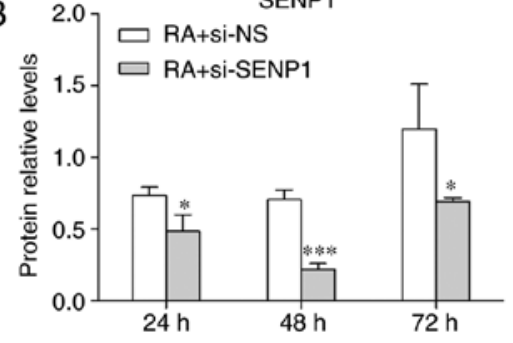

SPC

E kDa
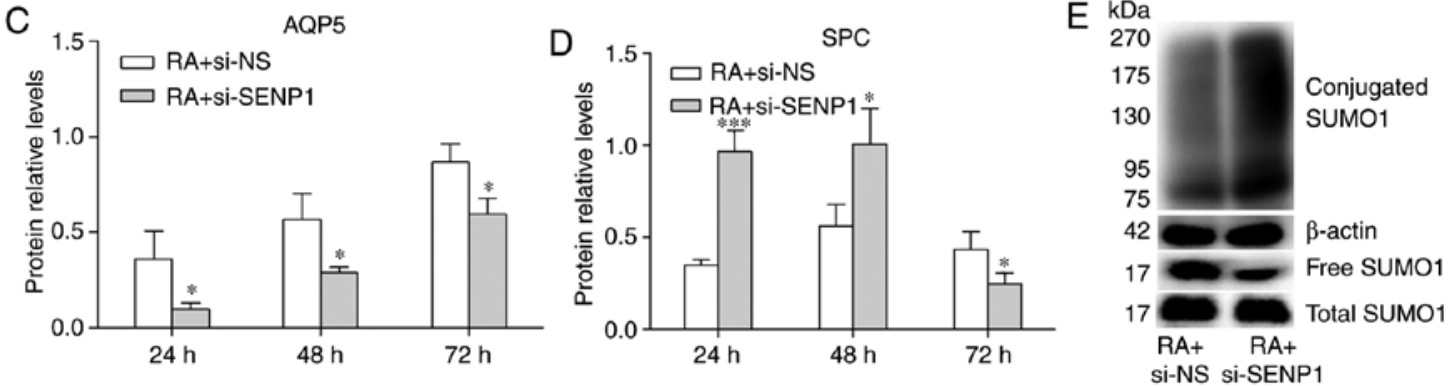

Figure 8. Effect of SENP1 inhibition on cell differentiation. siRNA were transfected into AT2 cells and cells were treated with RA. (A) Representative western blot of target protein is shown in. The expression levels of (B) SENP1, (C) AQP5, and (D) SP-C were semi-quantified. Values are presented as the mean \pm standard deviation from three independent experiments. ${ }^{*} \mathrm{P}<0.05,{ }^{* * * *} \mathrm{P}<0.001$ vs. RA + si-NS at the same time-point. (E) Protein levels of SUMO1 with SENP1 knockdown during the RA-induced differentiation were analyzed by western blotting. Protein extracts were prepared under denaturing conditions (sodium dodecyl sulfate). SP-C, surfactant protein C; AQP5, aquaporin-5; SENP1, SUMO specific protease 1; RA, retinoic acid; si, small interfering RNA; NS, non-specific.
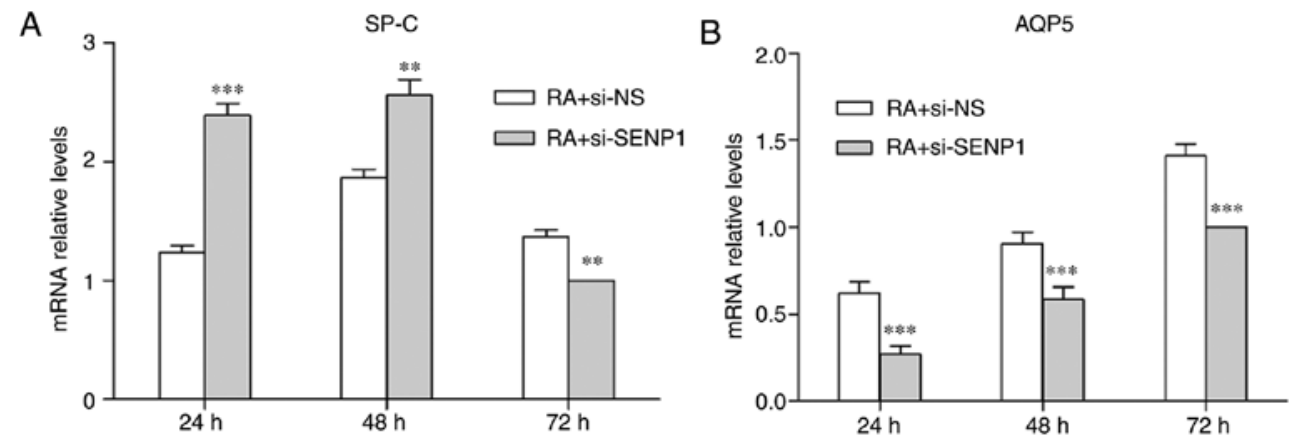

Figure 9. Relative expression levels of (A) SP-C and (B) AQP5 mRNA were detected by reverse transcription-quantitative polymerase chain reaction. Levels were normalized to the level at $72 \mathrm{~h}$, when cells were treated with RA + siSENP1. Values are presented as the mean \pm standard deviation of three independent experiments in duplicate. Statistical significance was determined using Student's t-test, ${ }^{* *} \mathrm{P}<0.01,{ }^{* * *} \mathrm{P}<0.001$ vs. RA + si-NS. SP-C, surfactant protein C; SENP1, SUMO specific protease 1; RA, retinoic acid; si, small interfering RNA; NS, non-specific; AQP5, aquaporin-5. 


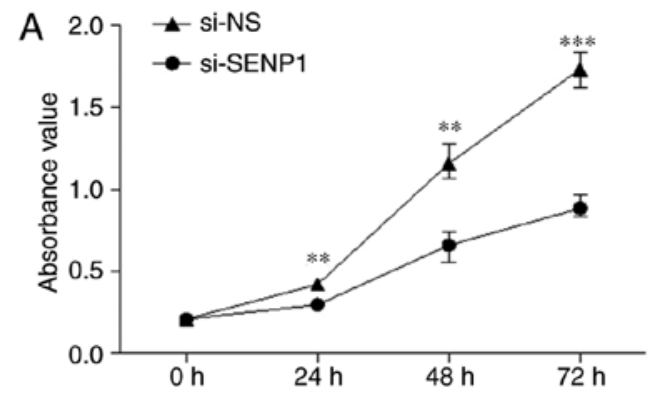

B
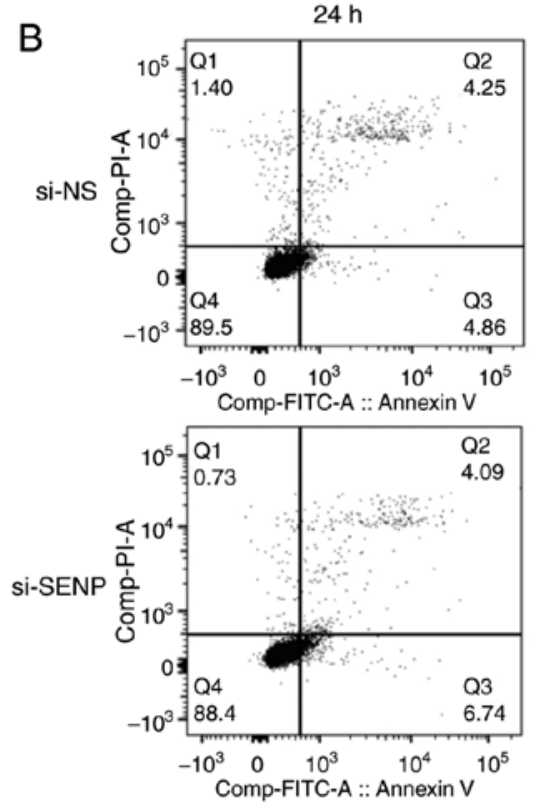

C

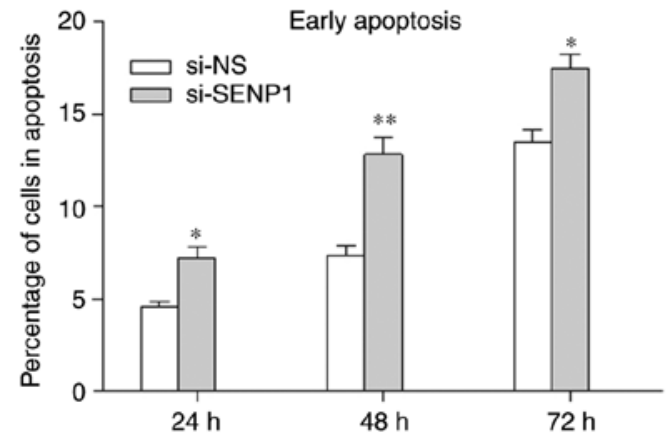

$48 \mathrm{~h}$
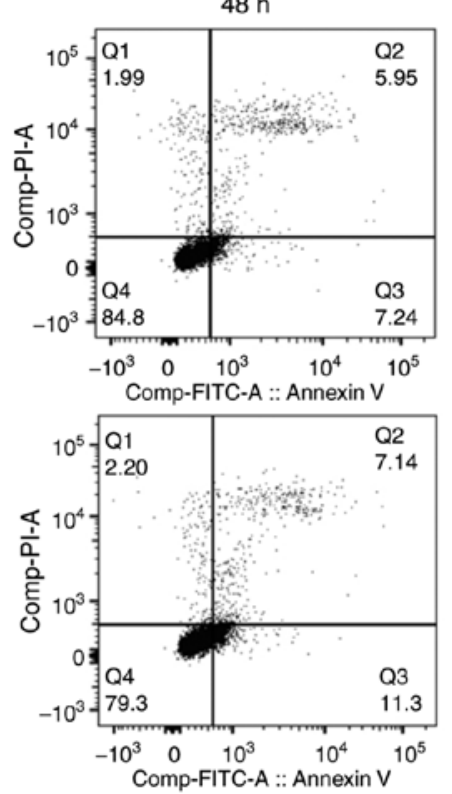
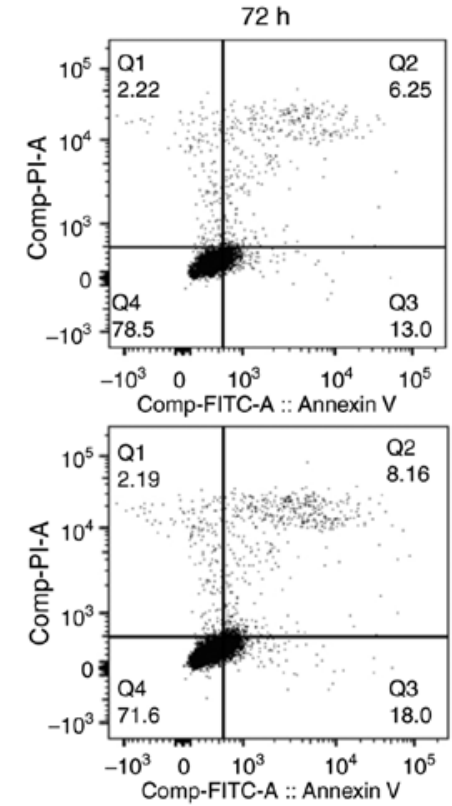

D

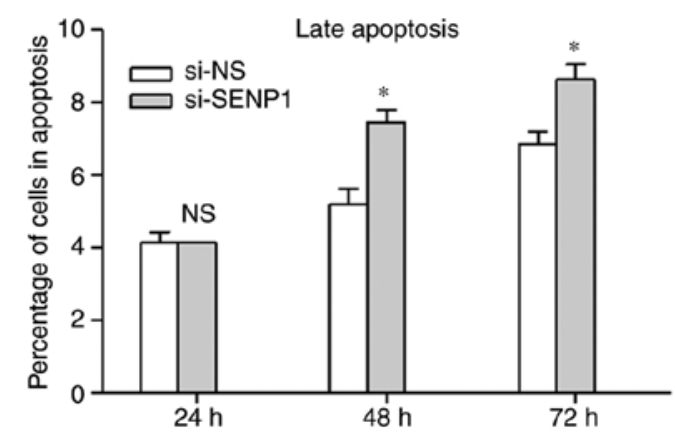

Figure 10. Effects of SENP1 inhibition on the cell proliferation and apoptosis of AT2 cells. (A) Cell proliferation was analyzed using CCK-8 assay following transfection of AT2 for 24, 48 and $72 \mathrm{~h}$. (B) Cell apoptosis was analyzed by flow cytometry when SENP1 knocked down in AT2 cells. Percentage of cells in (C) early and (D) late apoptosis. Statistical significance was determined using Student's t-test, ${ }^{*} \mathrm{P}<0.05,{ }^{* *} \mathrm{P}<0.01,{ }^{* * *} \mathrm{P}<0.001$ vs. si-SENP1. si, small interfering RNA; NS, non-specific; SENP1, SUMO specific protease 1; PI, propidium iodide; FITC, fluorescein isothiocyanate.

early and late apoptosis subgroups were higher compared with the si-NS group $(17.500 \pm 1.080$ vs. $13.467 \pm 1.034 \%$; and $8.637 \pm 0.585$ vs. $6.860 \pm 0.483 \%$, respectively) at $72 \mathrm{~h}$ after transfection. The significant decrease in cell apoptosis confirmed that the suppression of SENP1 caused AT2 cell death. Overall, the data indicate that SENP1 inhibition can reduce the proliferation of AT2 cells and promote apoptosis.

\section{Discussion}

Similar to human lung development, the lung development of rats is divided into five stages: Embryonic period, pseudoglandular period, canalicular period, saccular period and alveolar stage. At 1-4 days after birth, the rats are in the saccular stage of lung development. At days 5-14 (equivalent to 36 weeks of fetal lung development in human pregnancy), the rat lungs are in the alveolar phase (32). Therefore, neonatal rats can be used to investigate the association between the expression of SUMO1 and SENP1, and lung development.

Although free SUMO1 protein is differentially expressed during the lung development of neonatal rats, the mRNA levels of SUMO1 remain is stable in lung tissue at this stage. The protein levels of free and conjugated SUMO1 were also analyzed; with the total SUMO1 protein levels remaining 
unchanged. However, the expression level of free SUMO1 fluctuated and reached the highest level at post-natal day 4 . The level of conjugated SUMO1 was also variable, although its level was higher at day 14 than at day 4 . This data suggests that the observed changes in free SUMO are associated with the degree of protein SUMOylation. Sharma et al (24) reported that SENP1 is a major mediator of SUMO1 deconjugation and has a limited role in deSUMOylating SUMO2/3-modified proteins. On the basis of SUMO1 overexpression in Ca Ski cells, Yuasa and Saitoh (33) labeled SUMO1 protein with GFP in Ca Ski cells then added SENP1 catalytic domain into cell culture medium. The study revealed that the labeled SUMO1 was decreased significantly through the function of the SENP1 catalytic domain; the deSUMOylation of GFP directly demonstrated the effect of SENP1 on SUMO1 modification. In the current study, the expression of SENP1 was determined and revealing that the expression trend of SENP1 in at the gene and protein levels was consistent with that of free SUMO1 protein. Tissue morphological data indicated that that $\mathrm{P} 4$ is the most obvious period of alveolar formation. The alveolar morphology began to stabilize at P7-14. Consistent with these results, the expression of SENP1 decreased at P7 compared with P4, and expression was stable at P7-14. This indicates that SENP1 may regulate SUMO1 deconjugation to maintain the dynamic balance of protein SUMOylation and have an important role in lung development.

To further investigate the effect of SENP1 on protein SUMOylation and lung development in the present study, SENP1 was silenced in AT2 cells. AT2 is considered to be a stem cell of the alveolar epithelium $(3,5)$. In the process of normal cell renewal and repair, AT2 cells can differentiate into AT1 cells, or produce progeny AT2 via mitosis to maintain the cell population (34). SUMO1-conjugation was markedly increased in cells with SENP silencing compared with the control cells, indicating that depletion of SENP1 leads to disorder in SUMOylation and deSUMOylation. Previous studies have demonstrated that SUMOylation imbalance can lead to tumorigenesis, inflammatory diseases, DNA damage and impair cell differentiation $(10,14)$. Bronchopulmonary dysplasia (BPD) is a common serious respiratory disease in preterm infants. Compared with normal infants, the expression of free SUMO1 in the peripheral blood mononuclear cells of children with BPD is increased, while the expression of NAD-dependent protein deacetylase sirtuin-1 (SIRT1) and SUMOylated SIRT1 are decreased (35). These results suggest that the increase in free SUMO1 and decrease in SUMOylated SIRT1 may be associated with the occurrence of BPD. A previous study reported that the differentiation of multipotent stem cells into neurons was inhibited by overexpression of SUMO1 (25). It was speculated that SENP1 may have a function in the differentiation of AT2; thus, this was investigated by culturing AT2 cells in vitro. Compared with the control group, the SENP1 expression level was increased by adding RA to the medium to promote the differentiation of AT2. This confirmed that SENP1 was involved in the differentiation. Subsequently, RA was used to promote differentiation and expression of SENP1 in AT2 cells was inhibited using siRNA. Compared with the RA + si-NS group, the expression levels of AQP5 in the RA + si-SENP1 group were suppressed at the protein and mRNA level. The expression of SP-C was increased at 24 and $48 \mathrm{~h}$ after transfection, but decreased at $72 \mathrm{~h}$. These results demonstrate that despite the differentiation promoting effect of RA, the differentiation of AT2 was inhibited when SENP1 was suppressed. The inhibition of SENP1 in cells was confirmed to affect cell growth. The increase in SP-C was caused by inhibition of cell differentiation, while the decrease may be influenced by cell death. Further analysis demonstrated that silencing SENP1 in AT2 cells impairs cell proliferation and promotes apoptosis. This effect was most marked at $72 \mathrm{~h}$. The above results indicate that SENP1 is important for the differentiation of AT2s and can affect their growth. Numerous target proteins are regulated by the SUMOylation and deSUMOylation balance. Our previous study (17) demonstrated that SUMOylated C/EBP $\alpha$ was gradually decreased during lung differentiation and was negatively correlated with pulmonary surfactant secretion. This suggested that SUMO modification may be involved in C/EBP $\alpha$-mediated lung growth and differentiation. However, there has been limited research on the effects of protein SUMOylation and deSUMOylation on lung development and differentiation. The specifics of which proteins undergo SUMO modification and have roles in lung development and/or differentiation still require further clarification.

In conclusion, to the best of our knowledge, the current study is the first to demonstrate that SENP1 maintains the dynamic balance between protein SUMOylation and deSUMOylation during the alveolar period. In vitro experiments revealed that SENP1 regulates the proliferation and differentiation of AT2 cells via protein SUMOylation. The findings indicated that SENP1 is a key factor involved in normal lung development; however, whether SUMOylation of specific target proteins has a key role in the process requires further investigation.

\section{Acknowledgements}

Not applicable.

\section{Funding}

This work was financially supported by the National Natural Science Foundation of China (grant no. 81370746, 81741052) and the National Natural Science Foundation of Jiangsu Province (grant no. BK20161356).

\section{Availability of data and materials}

The datasets used and/or analyzed in this study are available from the corresponding author on reasonable request.

\section{Authors' contributions}

XQW conducted most of the experiments and was a major contributor in writing the paper. HYL, QXW and HMJ conceived the concept of the study. JYC and YZ cultivated cells and performed the PCR assay. HTZ performed the statistical analysis. All authors read and approved the final manuscript.

\section{Ethics approval and consent to participate}

The present study was approved by the Animal Center of Jiangsu University (Zhenjiang, China). 


\section{Patient consent for publication}

Not applicable.

\section{Competing interests}

The authors declare that they have no competing interests.

\section{References}

1. Warburton D and Bellusci S: The molecular genetics of lung morphogenesis and injury repair. Paediatr Respir Rev 5 (Suppl A) S283-S287, 2004

2. Claudio N and Morty RE: MicroRNA in late lung development and bronchopulmonary dysplasia: The need to demonstrate causality. Mol Cell Pediatr 3: 19, 2016.

3. Barkauskas CE, Cronce MJ, Rackley CR, Bowie EJ, Keene DR, Stripp BR, Randell SH, Noble PW and Hogan LM: Type 2 alveolar cells are stem cells in adult lung. J Clin Invest 123 : 3025-3036, 2013.

4. Adamson IY and Bowden DH: The type 2 cell as progenitor of alveolar epithelial regeneration: A cytodynamic study in mice after exposure to oxygen. Lab Invest 30: 35-42, 1974.

5. Adamson IY and Bowden DH: Derivation of type 1 epithelium from type 2 cells in the developing rat lung. Lab Invest 32 736-745, 1975

6. Siegel R, Naishadham D and Jemal A: Cancer statistics. CA Cancer J Clin 63: 11-30, 2013.

7. Lomelí $\mathrm{H}$ and Vázquez M: Emerging roles of the SUMO pathway in development. Cell Mol Life Sci 68: 4045-4064, 2011.

8. Garciadominguez M and Reyes JC: SUMO association with repressor complexes, emerging routes for transcriptional control. Biochim Biophys Acta 1789: 451-459, 2009.

9. Tempé D, Piechaczyk M and Bossis G: SUMO under stress Biochem Soc Trans 36: 874-878, 2008.

10. Kim JH and Baek SH: Emerging roles of desumoylating enzymes. Biochim Biophys Acta 1792: 155-162, 2009.

11. Saho E, Takuya A, Hiroshi A, Shunsuke K, Barnabas S, Yusuke Y, Akira M, Shunichi T and Dana B: The SUMO protease SENP1 is required for cohesion maintenance and mitotic arrest following spindle poison treatment. Biochem Biophys Res Commun 42 : 310-316, 2012.

12. Chen $\mathrm{CH}$, Chang CC, Lee TH, Luo M, Huang P, Liao P, Wei S, Li F, Chen R, Zhou XZ, et al: SENP1 deSUMOylates and regulates Pin1 protein activity and cellular function. Cancer Res 73 3951-3962, 2013.

13. Flotho A and Melchior F: Sumoylation: A regulatory protein modification in health and disease. Annu Rev Biochem 82: 357-385, 2013

14. Bawa-Khalfe T and Yeh ET: SUMO losing balance: SUMO proteases disrupt SUMO homeostasis to facilitate cancer development and progression. Genes Cancer 1: 748-752, 2010.

15. Nacerddine K, Lehembre F, Bhaumik M, Artus J, CohenTannoudji M, Babinet C, Pandolfi PP and Dejean A: The SUMO pathway is essential for nuclear integrity and chromosome segregation in mice. Dev Cell 9: 769-779, 2005.

16. Yamaguchi T, Sharma P, Athanasiou M, Kumar A, Yamada S and Kuehn MR: Mutation of SENP1/SuPr-2 reveals an essential role for desumoylation in mouse development. Mol Cell Biol 25: 5171-5182, 2005

17. Chen YD, Liu JY, Lu YM, Zhu HT, Tang W, Wang QX and Lu HY: Functional roles of C/EBP $\alpha$ and SUMO-modification in lung development. Int J Mol Med 40: 1037-1046, 2017.

18. Zhou F, Dai A, Fu D, Jiang Y, Tan X and Zhang X: SENP-1 enhances hypoxia-induced proliferation of rat pulmonary artery smooth muscle cells by regulating hypoxia-inducible factor- $1 \alpha$. Mol Med Rep 13: 3482-3490, 2016.
19. Jiang Y, Wang J, Tian H, Li G, Zhu H, Liu L, Hu R and Dai A: Increased SUMO-1 expression in response to hypoxia: Interaction with HIF-1 $\alpha$ in hypoxic pulmonary hypertension. Int J Mol Med 36: 271-281, 2015.

20. Pandey D, Nomura Y, Rossberg MC, Hori D, Bhatta A, Keceli G, Leucker T, Santhanam L, Shimoda LA, Berkowitz D and Romer L: Hypoxia triggers SENP1 (sentrin-specific protease 1) modulation of KLF15 (Kruppel-like factor 15) and transcriptional regulation of Arg2 (Arginase 2) in pulmonary endothelium. Arterioscler Thromb Vasc Biol 38: 913-926, 2018.

21. Wang RT, Zhi XY, Zhang Y and Zhang J: Inhibition of SENP1 induces radiosensitization in lung cancer cells. Exp Ther Med 6: 1054-1058, 2013

22. Livak KJ and Schmittgen TD: Analysis of relative gene expression data using real-time quantitative PCR and the 2(-Delta Delta C(T)) method. Methods 25: 402-408, 2001.

23. Gao RW, Kong XY, Zhu XX, Zhu GQ, Ma JS and Liu XX: Retinoic acid promotes primary fetal alveolar epithelial type II cell proliferation and differentiation to alveolar epithelial type I cells. In Vitro Cell Dev Biol Anim 51: 479-487, 2015.

24. Sharma P, Yamada S, Lualdi M, Dasso M and Kuehn MR: SENP1 is essential for desumoylating SUMO1-modified proteins but dispensable for SUMO2 and SUMO3 deconjugation in the mouse embryo. Cell Rep 3: 1640-1650, 2013.

25. Juarez-Vicente F, Luna-Pelaez N and Garcia-Dominguez M: The SUMO protease SENP7 is required for proper neuronal differentiation. Biochim Biophys Acta 1863: 1490-1498, 2016.

26. Besnard V, Nabeyrat E, Henrion-Caude A, Chadelat K, Perin L, Le Boucn Y and Clement A: Protective role of retinoic acid from antiproliferative action of TNF-alpha on lung epithelial cells. Am J Physiol Lung Cell Mol Physiol 282: L863-L871, 2002.

27. Londhe VA, Maisonet TM, Lopez B, Shin BC, Huynh J and Devaskar SU: Retinoic acid rescues alveolar hypoplasia in the calorierestricted developing rat lung. Am J Respir Cell Mol Biol 48: 179-187, 2013.

28. Evans MJ, Cabral LJ, Stephens RJ and Freeman G: Renewal of alveolar epithelium in the rat following exposure to $\mathrm{NO}_{2}$. Am J Pathol 70: 175-198, 1973.

29. Lu H, Chang L, Li W, Jiang N, Peng Q, Cai C and Liu J: Effects of hyperoxia on the dynamic expression of Aquaporin5 in premature rats lung development. J Huazhong Univ Sci Technolog Med Sci 27: 318-312, 2007.

30. George UM, Ashna U, Kumar SS and Nandkumar AM: Effect of tobacco extract on surfactant synthesis and its reversal by retinoic acid-role of cell-cell interactions in vitro. In Vitro Cell Dev Biol Anim 49: 260-269, 2013.

31. Nomura J, Horie I, Seto M, Nagai K, Hisatsune A, Miyata T and Isohama Y: All-trans retinoic acid increases expression of aquaporin-5 and plasma membrane water permeability via transactivation of Sp1 in mouse lung epithelial cells. Biochem Biophys Res Commun 351: 1048-1053, 2006.

32. Schittny JC: Development of the lung. Cell Tissue Res 367: 427-444, 2017

33. Yuasa E and Saitoh H: In situ SUMOylation and DeSUMOylation assays: fluorescent methods to visualize SUMOylation and DeSUMOylation in permeabilized cells. Methods Mol Biol 1475: 151-159, 2016.

34. Desai TJ, Brownfield DG and Krasnow MA: Alveolar progenitor and stem cells in lung development, renewal and cancer. Nature 507: 190-194, 2014.

35. Tan F, Dong W, Lei X, Li Q, Kang L, Zhao S and Zhang C: Attenuated SUMOylation of sirtuin 1 in premature neonates with bronchopulmonary dysplasia. Mol Med Rep 17: 1283-1288, 2018.

This work is licensed under a Creative Commons Attribution-NonCommercial-NoDerivatives 4.0 International (CC BY-NC-ND 4.0) License. 